\title{
LOS ELECTRODOS DE PASTA DE CARBONO EN EL ESTUDIO ELECTROQUíMICO DE MINERALES METÁLICOS
}

\author{
José L. Nava e Ignacio González* \\ Departamento de Química, Universidad Autónoma Metropolitana-Iztapalapa, Av. Sn Rafael Atlixco \# 186, \\ A.P. 55-534, C.P. 09340, México D.F., México
}

Recebido em 29/6/04; aceito em 8/11/04; publicado na web em 28/2/05

\begin{abstract}
THE ROLE OF THE CARBON PASTE ELECTRODES IN THE ELECTROCHEMICAL STUDY OF METALLIC MINERALS. This paper shows the applicability of the carbon paste electrode-mineral (CPE-mineral) to study the dissolution mechanisms of minerals in powder form and in flotation concentrates. A potentiodynamic strategy to find the dissolution mechanism of galena $(\mathrm{PbS})$ is presented. In this way, minerals less studied such as orpiment $\left(\mathrm{As}_{2} \mathrm{~S}_{3}\right)$ and realgar $\left(\mathrm{As}_{2} \mathrm{~S}_{2}\right)$ are investigated. The electrochemical activity of a more complicated mineral such as sphalerite ( $\mathrm{ZnS})$, containing 12.3 and $0.43 \%$ of iron in solid solution, is discussed. The mechanism of a complex zinc concentrate (containing $63.4 \% \mathrm{ZnS}, 20.1 \% \mathrm{FeS}_{2}, 5 \% \mathrm{CuFeS}_{2}, 0.33 \% \mathrm{PbS}, 0.45 \%$ $\mathrm{Cu}_{12} \mathrm{Sb}_{4} \mathrm{~S}_{13}$ and $0.4 \% \mathrm{FeAsS}$ ) is described. Finally, an electrochemical method for the detection of the different leachable and refractory silver phases (contained in two mineral concentrates) is presented. This paper reviews the power of the use of CPEmineral coupled to electrochemical techniques in hydrometallurgy.
\end{abstract}

Keywords: CPE-mineral; dissolution mechanisms; electrochemical techniques.

\section{INTRODUCCIÓN}

Los sulfuros minerales son la principal fuente para la obtención de metales base, siendo los procesos pirometalúrgicos los que han sido tradicionalmente empleados para el procesamiento de estos minerales. Sin embargo, la emisión de $\mathrm{SO}_{2 \text { (gas) }}$ y de partículas sólidas suspendidas en el aire, durante la combustión, ha llevado a la búsqueda de procesos limpios, siendo la ruta hidrometalúrgica la que ha recibido especial atención ${ }^{1}$.

Los procesos de disolución oxidativa de muchos minerales (empleando oxidantes como $\mathrm{Fe}(\mathrm{III}), \mathrm{O}_{2}$, entre otros), han presentado algunos problemas que han impedido tener eficiencias satisfactorias en la aplicación industrial ${ }^{1}$. Es importante mencionar que las reacciones que toman lugar en los procesos hidrometalúrgicos son de naturaleza electroquímica y por lo tanto, su entendimiento es indispensable para la optimización de estos procesos.

En el estudio electroquímico de minerales, se pueden emplear cristales de mineral como electrodos de prueba. No obstante, algunos minerales presentan ciertas propiedades semiconductoras que complican su caracterización. Por otro lado, la reproducibilidad experimental que estos electrodos proporcionan no es confiable, dado que la renovación de la superficie, para cada nueva experiencia es complicada.

Los electrodos de pasta de carbono-mineral (CPE-mineral), donde el mineral pulverizado es mezclado con polvo de grafito, han mostrado su conveniencia en el estudio de mecanismos de minerales. Los CPE-mineral, pueden utilizar un aglomerante conductor, que puede ser $\mathrm{H}_{2} \mathrm{SO}_{4}$ o bien $\mathrm{HCl}^{2,3}$. Por otro lado, los CPEmineral pueden ser preparados con aglomerantes no conductores ya sea usando aceites orgánicos, o bien aceites minerales, los cuales suelen ser hidrofóbicos ${ }^{4,5}$, permitiendo que las reacciones electroquímicas sólo tomen lugar en la interfase electrodo-

*e-mail: igm@xanum.uam.mx electrolito. Esto último es diferente para los CPE-mineral con aglomerante conductor, donde las reacciones electroquímicas también toman lugar dentro del electrodo ${ }^{2,3}$. Por lo ya mencionado, los CPE-mineral con aglomerante no conductor han mostrado su conveniencia en el estudio de minerales ${ }^{4-6}$.

Es importante mencionar que la sensibilidad de los CPE-mineral es alta debido a sus bajas corrientes residuales; además, ciertos minerales poco conductores (de tipo $n$ ) pueden ser estudiados empleando la técnica de CPE-mineral, lo cual no se puede lograr empleando electrodos sólidos ${ }^{6}$. Esto último es debido a que un mineral poco conductor homogéneamente disperso en una matriz de grafito ve mejorada su conductividad debida a un fenómeno de percolación ${ }^{6}$.

En esta comunicación se muestra la conveniencia de emplear CPE-mineral con aglomerante no conductor (aceite de silicón) para encontrar mecanismos de disolución de ciertos minerales altamente puros, o bien provenientes de procesos de flotación. Los estudios electroquímicos mostrados en este artículo son presentados de acuerdo a la complejidad que presenta cada mineral, y son una recopilación de información encontrada por el grupo de Electroquímica de la UAM-I.

Se presenta el estudio del mecanismo de disolución de un concentrado de galena ( $\mathrm{PbS}$, altamente puro) proveniente de un proceso de flotación. Este estudio es presentado de manera sistemática, con el propósito de mostrar cómo a través de una estrategia voltamperométrica fue posible encontrar su mecanismo de electrodisolución. El segundo estudio mostrado fue sobre dos minerales poco estudiados como son el rejalgar $\left(\mathrm{As}_{2} \mathrm{~S}_{2}\right)$ y el oropimente $\left(\mathrm{As}_{2} \mathrm{~S}_{3}\right)$. En este segundo estudio sólo se discuten algunos voltamperogramas que ilustran el mecanismo de ambos minerales. El tercer estudio tuvo como objetivo mostrar la actividad electroquímica de un mineral poco conductor (esfalerita, $\mathrm{ZnS}$ ), analizando dos muestras que contenían 13,8 y $0,43 \%$ de Fe como solución sólida. Por otro lado, se muestra una estrategia electroquímica más elaborada que permitió encontrar el mecanismo de disolución de un concentrado complejo de cinc $(63,4 \% \mathrm{ZnS}, 20,1 \%$ 
$\mathrm{FeS}_{2}, 5 \% \mathrm{CuFeS}_{2}, 0,33 \% \mathrm{PbS}, 0,45 \% \mathrm{Cu}_{12} \mathrm{Sb}_{4} \mathrm{~S}_{13}$ y $\left.0,4 \% \mathrm{FeAsS}\right)$. Finalmente, se muestra un método electroquímico que permite identificar las propiedades refractarias a la lixiviación de diferentes fases de plata contenidas en dos concentrados minerales. Los electrodos de referencia empleados para el estudio electroquímico de cada mineral, en algunos casos fueron diferentes, ya que estos consideraron al electrolito y al mineral. Sin embargo, para hacer un estudio comparativo, en esta revisión, todos los potenciales son mostrados con respecto al electrodo de calomelanos (SCE, por sus siglas en inglés).

\section{Galena en medio de ácido perclórico}

A continuación se muestra un resumen de una metodología que permitió encontrar el mecanismo de un mineral muy estudiado (galena) empleando CPE-galena (80:20\% peso), en medio de ácido perclórico ${ }^{7}$.

En la Figura 1a,b, se muestran dos voltamperogramas típicos obtenidos sobre un CPE-galena sin agitación de electrolito, cuyo barrido de potencial fue iniciado en dirección negativa y positiva, respectivamente, a partir del potencial de circuito abierto.

La comparación de los voltamperogramas mostrados en la Figura 1a,b, indicaron que los procesos (B') y (C'), están relacionados con los procesos oxidativos en (A'), mientras que (D) y (D') corresponden a la reducción de galena. A continuación se muestra un estudio voltamperométrico variando los potenciales de inversión catódicos $\left(\mathrm{E}_{\lambda_{-}}\right)$y anódicos $\left(\mathrm{E}_{\lambda_{+}}\right)$.

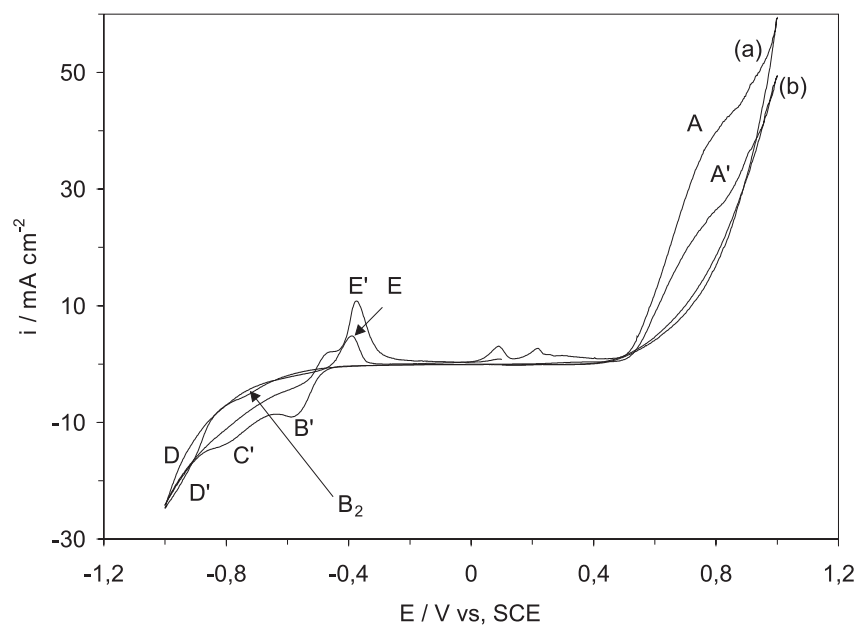

Figura 1. Voltamperogramas típicos obtenidos sobre CPE-galena (80:20\% peso) en 1,0 $\mathrm{M} \mathrm{HClO}_{4},\left(v=100 \mathrm{mV} \mathrm{s} \mathrm{s}^{-1}\right)$. El área del CPE-galena fue de $0,0314 \mathrm{~cm}^{2}$. El barrido de potencial fue iniciado en la dirección: (a) negativa y (b) positiva. Reimpresa da ref. 7, con permiso de Elsevier

\section{Procesos de reducción de galena}

Para identificar los procesos de reducción de galena, se realizó un estudio voltamperométrico en la dirección negativa cambiando el potencial de inversión negativo, $\mathrm{E}_{\lambda,}$, (Figura 2). El intervalo de estudio estuvo comprendido entre $-0,8 \leq \mathrm{E}_{\lambda_{-}} \leq-0,6 \mathrm{~V}$, con incrementos de $0,1 \mathrm{~V}$.

De acuerdo a la Figura 2a, cuando $\mathrm{E}_{\lambda-}=-0,6 \mathrm{~V}$, apareció una corriente de reducción, la cual no tiene asociado ningún proceso de oxidación. Cuando $\mathrm{E}_{\lambda-}=-0,7 \mathrm{~V}$, Figura $2 \mathrm{~b}$, una preonda $\left(\mathrm{B}_{2}\right)$ que antecede a los procesos asociados a (D), es perceptible. Además, $\left(\mathrm{B}_{2}\right)$, está asociada con un pequeño pico ancho de oxidación (E). A un $E_{\lambda_{-}}=-0,80 \mathrm{~V}$, Figura $2 \mathrm{c}$, se detectó un incremento considerable

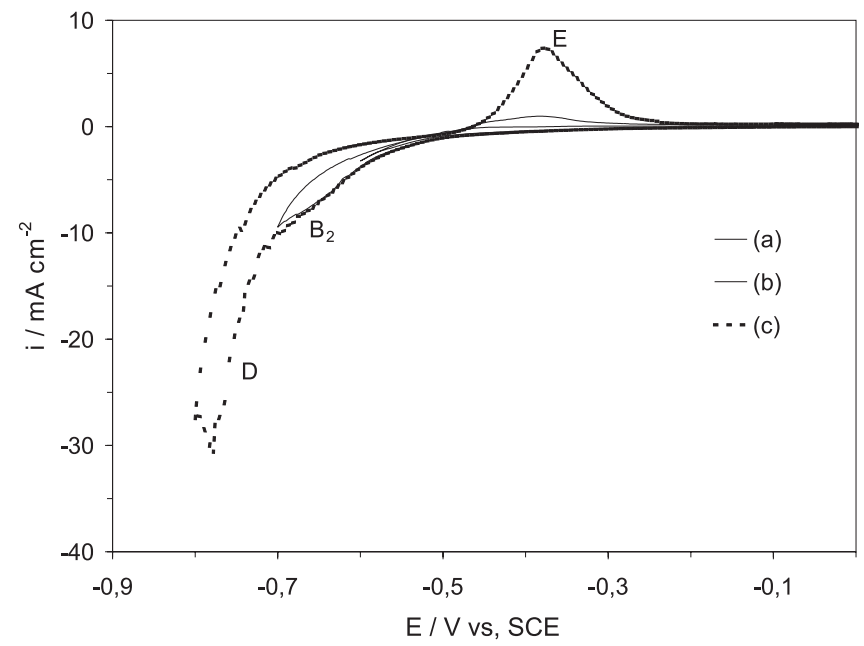

Figura 2. Voltamperogramas típicos de CPE-galena (80:20\% peso) en 1,0 M $\mathrm{HClO}_{4},\left(v=100 \mathrm{mV} \mathrm{s}^{-1}\right)$. El área del CPE-galena fue de $0,0314 \mathrm{~cm}^{2}$. El barrido de potencial fue iniciado en la dirección negativa a $100 \mathrm{mV} \mathrm{s}^{-1}$. Los potenciales de inversión, $E_{\lambda}$ son: (a) -0,60, (b) -0,70 y (c) -0,80 V. Sin agitación de electrolito. Reimpresa da ref. 7 con permiso de Elsevier

en corriente, asociado al proceso (D), y cuando se invirtió el barrido de potencial, se detectó un pico ancho (E) mejor definido.

Experimentos cuando el electrolito fue agitado ${ }^{7}$ indicaron que las especies reducidas en $\left(\mathrm{B}_{2}\right)$ son solubles y se encuentran en la interfase en baja concentración debido a que este proceso no fue detectado. Es importante mencionar que el proceso (D) y su correspondiente oxidación (E) fueron detectados con agitación. Esto último sugiere que el proceso que toma lugar en (D) corresponde a la reducción directa de galena:

$\mathrm{PbS}+2 \mathrm{H}^{+}+2 \mathrm{e}^{-} \leftrightarrow \mathrm{Pb}^{0}+\mathrm{H}_{2} \mathrm{~S}$

$\mathrm{E}^{*}=-0,595 \mathrm{~V}$ vs. SCE

El potencial de equilibrio, E*, relacionado con la ecuación (1) fue calculado tomando en cuenta el correspondiente potencial normal, $\mathrm{E}^{0}$, para la reacción $(1)^{8}$ y considerando una concentración $10^{-6} \mathrm{M}$ para las especies formadas en la interfase. Esta consideración es frecuentemente usada cuando se trata de la corrosión de metales o la lixiviación de concentrados minerales 9.

De esta manera, el proceso de oxidación asociado al pico (E), corresponde a la oxidación del $\mathrm{Pb}(0)$ a $\mathrm{Pb}(\mathrm{II})$, inversa de la Ecuación 2:

$\mathrm{Pb}^{2+}+2 \mathrm{e}^{-} \rightarrow \mathrm{Pb}^{0}$

$\mathrm{E}^{*}=-0,544 \mathrm{~V}$ vs. SCE

Por otro lado, debido a que el pico $\left(\mathrm{B}_{2}\right)$ desapareció con la agitación, $\mathrm{Nava}^{7}$ y colaboradores propusieron que el proceso de reducción asociado al pico $\left(\mathrm{B}_{2}\right)$, mostrado en la Figura $2 b$, corresponde a la reducción de $\mathrm{Pb}$ (II) proveniente de un proceso de disolución no oxidativo de galena (durante el tiempo de inmersión del CPE-galena en el electrolito):

$\mathrm{PbS}+2 \mathrm{H}^{+} \leftrightarrow \mathrm{Pb}^{2+}+\mathrm{H}_{2} \mathrm{~S}_{(\mathrm{aq})}$

Esta reacción es termodinámicamente posible a condiciones de $\mathrm{pH}$ muy ácidas ${ }^{10}$,

Del voltamperograma mostrado en la Figura $2 \mathrm{c}$ a $\mathrm{E}_{\lambda-}=-0,8 \mathrm{~V}$, la relación entre la carga anódica del proceso (E) y la carga catódica, 
fue $\mathrm{Q}_{\mathrm{E}} / \mathrm{Q}_{\mathrm{c}}=0,162$, sugiriendo que además de los procesos de reducción descritos por las ecuaciones (1) y (2), se están reduciendo los protones del medio.

\section{Procesos de oxidación de galena}

Con el fin de acotar la zona de potencial donde la galena es oxidada se llevó a cabo un estudio voltamperométrico, cambiando los potenciales anódicos de inversión $\left(\mathrm{E}_{\lambda_{+}}\right)$. Estos $\left(\mathrm{E}_{\lambda_{+}}\right)$estuvieron comprendidos entre $0,50 \leq \mathrm{E}_{\lambda+} \leq 0,70 \mathrm{~V}$. El límite catódico $\left(\mathrm{E}_{\lambda}\right)$ se mantuvo constante en $-0,65 \mathrm{~V}$, ya que a este potencial no se reduce la galena, proceso (D').

En la Figura $3 b-d$ se presentan tres voltamperogramas a $E_{\lambda_{+}}$de $0,54,0,58$ y $0,62 \mathrm{~V}$, respectivamente. Estos voltamperogramas fueron comparados con uno iniciado negativamente, Figura 3a. Las corrientes anódicas asociadas a los procesos en (A') crecen como función de $\mathrm{E}_{\lambda+}$, al igual que las corrientes en (B') y en (E'); estas corrientes fueron mayores que la obtenida iniciando el barrido de potencial negativamente, Figura $3 \mathrm{a}$.

Voltamperogramas similares a los mostrados en la Figura 3, trazados con agitación de electrolito ${ }^{7}$ mostraron que las corrientes asociadas en (A') también incrementadas como función de $\mathrm{E}_{\lambda+}$; sin embargo, éstas fueron menores que las obtenidas sin agitación y los procesos (B') y (E') desaparecieron. De esta manera, Nava y colaboradores $^{7}$ sugirieron que las especies que se reducen en (B') son solubles.

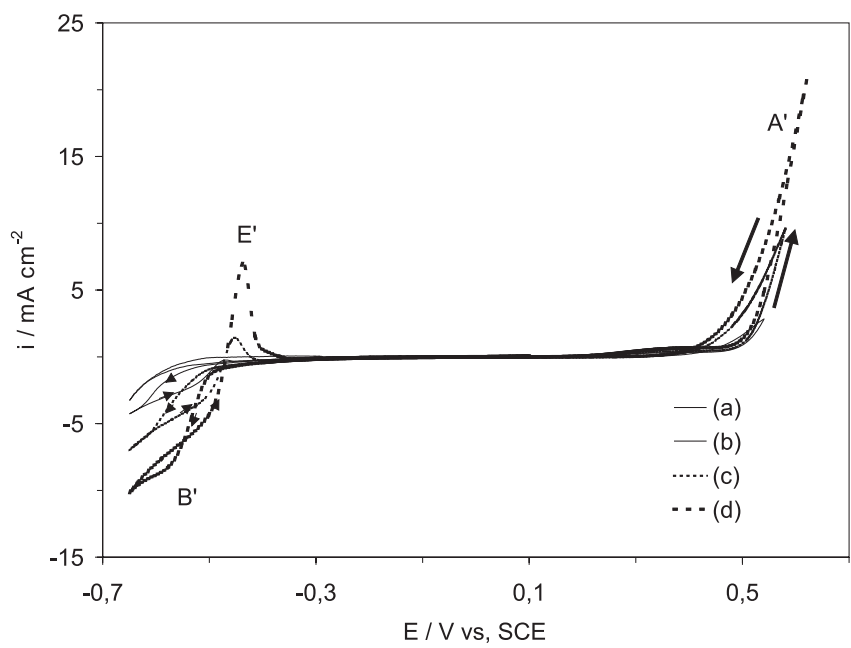

Figura 3. Voltamperogramas típicos sobre CPE-galena (80:20\% peso) en 1,0 $\mathrm{M} \mathrm{HClO}_{4},\left(v=100 \mathrm{mV} \mathrm{s}^{-1}\right)$. El área del CPE-galena fue de $0,0314 \mathrm{~cm}^{2} . \mathrm{El}$ barrido de potencial fue fijado en-0,65 V. El barrido de potencial fue iniciado en la dirección positiva y el pulso de potencial positivo $\left(E_{\lambda^{+}}\right)$, fue variado: (b) 0,54, (c) 0,58 y (d) 0,62 V. Con el propósito de comparar los procesos de reducción, en (a) se muestra un voltamperograma cuyo barrido inició en la dirección negativa. Sin agitación de electrolito. Reimpresa da ref. 7, con permiso de Elsevier

Estos mismos autores evaluaron las cargas asociadas a los procesos en (A'), (B') y (E') de los voltamperogramas obtenidos a los diferentes $\left(E_{\lambda_{+}}\right)$comprendidos entre $0,50 \leq E_{1+} \leq 0,70 \mathrm{~V}$, con y sin agitación. En la Figura 4a-d, se muestran las cargas asociadas a $\mathrm{Q}_{\mathrm{A}}, \mathrm{Q}_{\mathrm{B}}, \mathrm{y}_{\mathrm{E}}$, como función de $\mathrm{E}_{\lambda+}$ sin y con agitación de electrolito.

Comparando la tendencia de las cargas anódicas, $\mathrm{Q}_{\mathrm{A}}$, se observa que sin agitación, ésta se ve favorecida respecto a la obtenida con agitación, a $\mathrm{E}_{\lambda_{+}}>0,54 \mathrm{~V}$, Figura $4 \mathrm{a}$ y d, respectivamente. Por otro lado, al comparar la carga catódica, $\mathrm{Q}_{\mathrm{B}}$, con $\mathrm{Q}_{\mathrm{A}}$, sin agitación, Figura $4 \mathrm{~b}$ y a, se observa que guardan una relación $2: 1$ a $\mathrm{E}_{\lambda+} \leq 0,56$

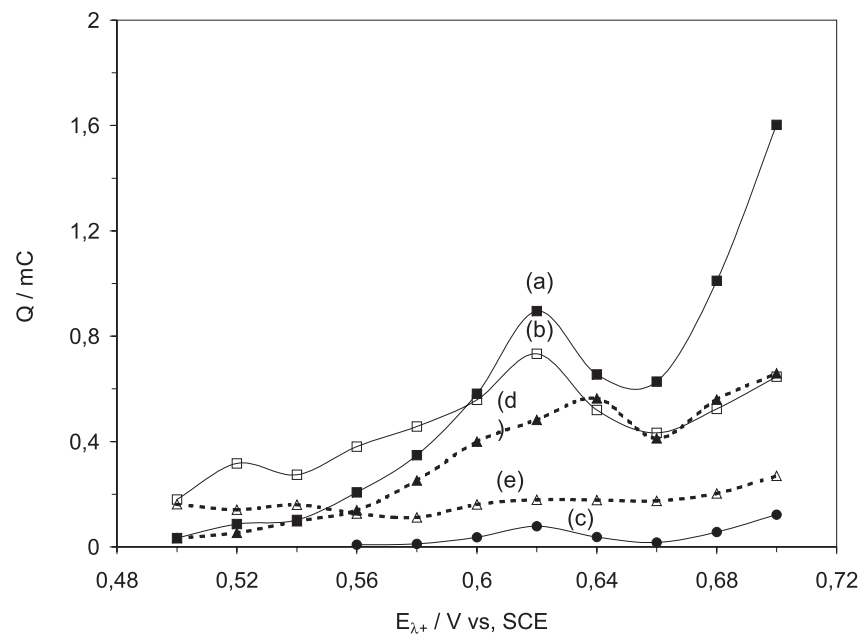

Figura 4. Variación de la carga voltamperométrica, $Q$, con el pulso potencial de inversión positivo $E_{\lambda_{+}}$. Las cargas fueron evaluadas de los voltamperogramas similares a los mostrados en la Figura 3. Las cargas asociadas a los procesos sin agitación de electrolito son: (a) $Q_{A}$, (b) $Q_{B}$, (c) $Q_{E^{*}}$. Con agitación: (d) $Q_{A^{\prime}}$ y (e) $Q_{B}$. Reimpresa da ref. 7, con permiso de Elsevier

$\mathrm{V}$; sin embargo, a partir de este potencial la relación disminuye hasta volverse 1:1 en $\mathrm{E}_{\lambda+}=0,60 \mathrm{~V}$. Pasando este potencial se invierte la proporción, por lo tanto, cualitativamente se pueden describir dos zonas para la oxidación de galena: La primera a $\mathrm{E}_{\lambda_{+}} \leq 0,60 \mathrm{~V}$, donde la oxidación de galena es descrita como:

$\mathrm{PbS} \rightarrow \mathrm{S}+\mathrm{Pb}^{2+}+2 \mathrm{e}$

$\mathrm{E}^{*}=-0,077 \mathrm{~V}$ vs. SCE

Cabe mencionar que la oxidación de sulfuros minerales requiere de altos sobrepotenciales relativos al de equilibrio ${ }^{7,10,11}$.

La segunda oxidación de galena se da a $\mathrm{E}_{\lambda_{+}} \geq 0,62 \mathrm{~V}$ :

$2 \mathrm{PbS}+3 \mathrm{H}_{2} \mathrm{O} \rightarrow 2 \mathrm{~Pb}^{2+}+\mathrm{S}_{2} \mathrm{O}_{3}{ }^{2-}+6 \mathrm{H}^{+}+8 \mathrm{e}^{-}$

$\mathrm{E}^{*}=0,067 \mathrm{~V}$ vs. SCE

Considerando que los tiosulfatos comienzan a formarse a partir de $0,6 \mathrm{~V}$, este alto sobrepotencial permite que el $\mathrm{S}_{2} \mathrm{O}_{3}{ }^{2-}$ formado en la interfase, sea oxidado a $\mathrm{SO}_{4}^{2-}$, dado que el $\mathrm{E}^{*}$ de la Ecuación (6), es menos positivo al requerido en la Eecuación (5).

$\mathrm{S}_{2} \mathrm{O}_{3}{ }^{2-}+5 \mathrm{H}_{2} \mathrm{O} \rightarrow 2 \mathrm{SO}_{4}{ }^{2-}+10 \mathrm{H}^{+}+8 \mathrm{e}$

$\mathrm{E}^{*}=-0,004 \mathrm{~V}$ vs. SCE

Es importante mencionar que la $\mathrm{Q}_{\mathrm{B}}$, para $\mathrm{E}_{\lambda_{+}} \leq 0,58 \mathrm{~V}$, corresponde casi al doble que $\mathrm{Q}_{\mathrm{A}}$, sin agitación (Figura 4a-b). Por lo tanto, se puede establecer que el proceso de reducción en ( $\left.\mathrm{B}^{\prime}\right)$, a $\mathrm{E}_{\lambda_{+}}<0,60 \mathrm{~V}$, involucra tanto la reducción de $\mathrm{Pb}(\mathrm{II})$ a $\mathrm{Pb}(0)$ de acuerdo a la Ecuación (2), como a la reducción de azufre:

$\mathrm{S}^{0}+2 \mathrm{H}^{+}+2 \mathrm{e}^{-} \rightarrow \mathrm{H}_{2} \mathrm{~S}(\mathrm{aq})$

$\mathrm{E}^{*}=-0,097 \mathrm{~V}$ vs. SCE

Esta reducción de azufre elemental se detectó en las curvas mostradas en la Figura $3 \mathrm{~b}-\mathrm{c}$, para los procesos de reducción asociados en (B'), donde a $\mathrm{E}_{\lambda+}$ de 0,54 y 0,58 se manifestó como un proceso de activación. De esta manera se explica la relación 2:1 para $\mathrm{Q}_{\mathrm{B}}: \mathrm{Q}_{\mathrm{A}}$, sin agitación a $\mathrm{E}_{\lambda+}<0,60 \mathrm{~V}$ (ver Figura $4 \mathrm{a}-\mathrm{b}$ ), dado que la carga asociada a la oxidación de galena, $\mathrm{Q}_{\mathrm{A}}$, involucra $2 \mathrm{e}$, 
Eecuación (4), mientras que $\mathrm{Q}_{\mathrm{B}}$, que corresponde a las reacciones descritas por las Ecuaciones (2) y (7), involucran entre ambas $4 \mathrm{e}^{-}$.

Dado que $\mathrm{Q}$, fue siempre mayor sin agitación que con agitación entre $0,56 \leq \mathrm{E}_{\lambda_{+}} \leq 0,60 \mathrm{~V}$, Figura. 4a y d, Nava y colaboradores $^{7}$ discuten este hecho diciendo que la presencia de plomo en la interfase le proporciona mayor porosidad a la capa de azufre elemental, haciendo más cuantitativa la electrodisolución.

En este resumen sobre galena se mostró una estrategia voltampeométrica que permitió encontrar los mecanismos de oxidación-reducción de galena.

\section{Estudio electroquímico de oropimente $\left(\mathrm{As}_{2} \mathrm{~S}_{3}\right)$ y rejalgar $\left(\mathrm{As}_{2} \mathrm{~S}_{2}\right)$ en el medio de cultivo (M2) en ausencia de acidithiobacillus ferrooxidans}

El oropimente $\left(\mathrm{As}_{2} \mathrm{~S}_{3}\right)$ y rejalgar $\left(\mathrm{As}_{2} \mathrm{~S}_{2}\right)$ son dos minerales sulfurosos de fácil alteración, que se encuentran asociados a la arsenopirita (FeAsS $)$ y pirita $\left(\mathrm{FeS}_{2}\right)$. En esta comunicación se toman los resultados obtenidos por Lázaro y colaboradores ${ }^{12}$, siendo de los pocos estudios electroquímicos realizados para estos dos minerales. Este estudio fue llevado a cabo en un medio de cultivo (M2), empleado para una bacteria acidofílica autotrófica conocida como acidithiobacillus ferrooxidans. Es importante mencionar que todos los experimentos desarrollados por Lázaro y colaboradores ${ }^{12}$ fueron en ausencia de acidithiobacillus ferrooxidans. Estos autores tuvieron la necesidad de llevar a cabo un estudio electroquímico sobre $\left(\mathrm{As}_{2} \mathrm{~S}_{3}\right)$ y $\left(\mathrm{As}_{2} \mathrm{~S}_{2}\right)$ en el medio (M2) para entender a la oxidación de (FeAsS), dado que la oxidación de arsenopirita en este medio (M2) forma $\left(\mathrm{As}_{2} \mathrm{~S}_{3}\right)$ y $\left(\mathrm{As}_{2} \mathrm{~S}_{2}\right)$ como productos intermedia$\operatorname{rios}^{13}$. Los detalles de la composición del medio (M2) están en el artículo publicado por Lázaro y colaboradores ${ }^{12}$.

En la Figura 5, se muestran dos voltamperogramas típicos sobre $\mathrm{CPE}-\mathrm{As}_{2} \mathrm{~S}_{3}$ en (M2). El barrido de potencial se inició en direc-
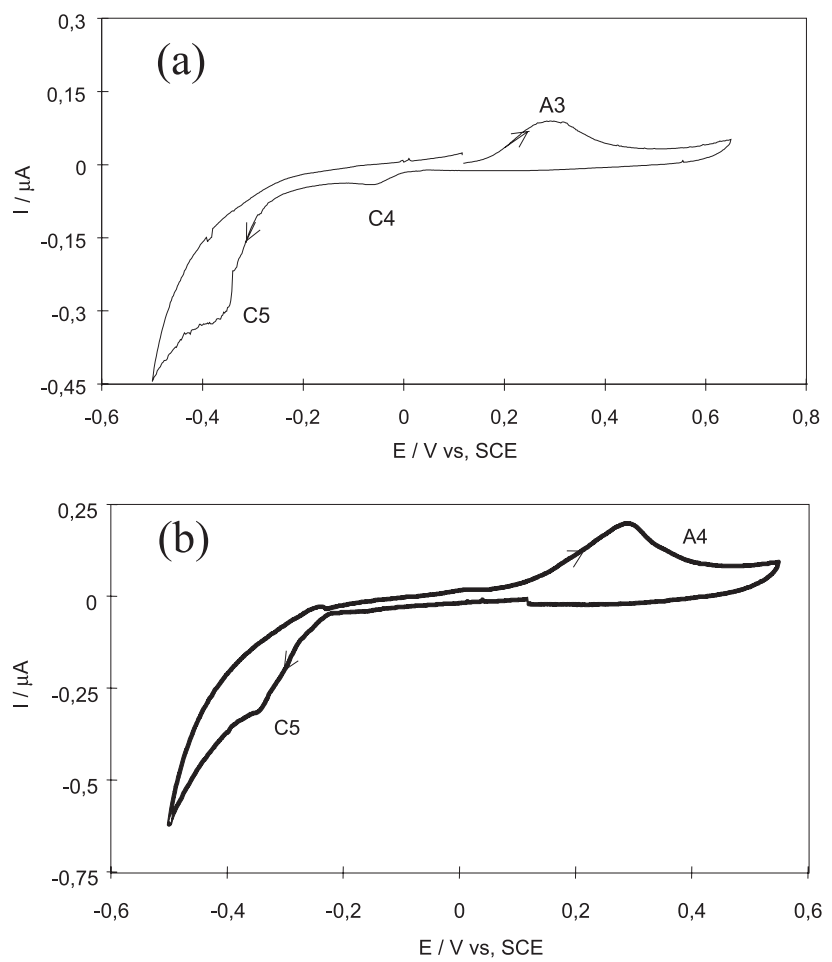

Figura 5. Voltamperograma típico de CPE-As $S_{3}(50: 50 \%$ peso) en el medio de cultivo M2, $v=20 \mathrm{mV} \mathrm{s}^{-1}$. El área del CPE-As $S_{2}$ fue de $0,0314 \mathrm{~cm}^{2}$. Barrido de potencial iniciado en dirección: (a) anódica y (b) catódica. Reimpresa da ref. 12, con permiso de The Electrochemical Society, Inc. ción anódica (Figura 5a) y catódica (Figura 5b), a una velocidad de barrido de $20 \mathrm{mV} \mathrm{s}^{-1}$. La comparación de ambos voltamperogramas indican que los procesos $(\mathbf{C 4})$ y $(\mathbf{C 5})$ corresponden a la reducción de los productos de oxidación formados en (A3). Es importante mencionar que cuando el barrido fue iniciado en la dirección negativa sólo se detectó el proceso (C5), el cual fue menos definido. Cuando se invirtió el barrido se observó el pico (A4), el cual fue más amplio que (A3), Figura 5a,b.

Lázaro y colaboradores ${ }^{12}$ realizaron un amplio estudio variando los potenciales anódicos $\left(\mathrm{E}_{\lambda_{+}}\right)$y catódicos $\left(\mathrm{E}_{\lambda}\right)$, y de este estudio estos autores proponen el siguiente mecanismo:

Proceso A3

$\mathrm{As}_{2} \mathrm{~S}_{3}+18 \mathrm{H}_{2} \mathrm{O} \rightarrow 2 \mathrm{H}_{3} \mathrm{AsO}_{3}+3 \mathrm{HSO}_{4}^{-}+27 \mathrm{H}^{+}+24 \mathrm{e}^{-}$

seguido de la oxidación del ácido arsenioso $\left(\mathrm{H}_{3} \mathrm{AsO}_{3}\right)$ a ácido arsénico $\left(\mathrm{H}_{3} \mathrm{AsO}_{4}\right)$

$\mathrm{H}_{3} \mathrm{AsO}_{3}+\mathrm{H}_{2} \mathrm{O} \rightarrow \mathrm{H}_{3} \mathrm{AsO}_{4}+2 \mathrm{H}^{+}+2 \mathrm{e}^{-}$

Proceso (C4):

Reacción inversa de la Ecuación (9)

Proceso (C5):

corresponde a la reducción del oropimente en dos etapas:

$\mathrm{As}_{2} \mathrm{~S}_{3}+2 \mathrm{H}^{+}+2 \mathrm{e}^{-} \rightarrow \mathrm{As}_{2} \mathrm{~S}_{2}+\mathrm{H}_{2} \mathrm{~S}$

$\mathrm{As}_{2} \mathrm{~S}_{2}+4 \mathrm{H}^{+}+4 \mathrm{e}^{-} \rightarrow 2 \mathrm{As}+2 \mathrm{H}_{2} \mathrm{~S}$

En la Figura 6, se presentan dos voltamperogramas similares a los mostrados en la Figura 5, sólo que ahora sobre CPE-rejalgar. La comparación de ambos voltamperogramas permitió observar
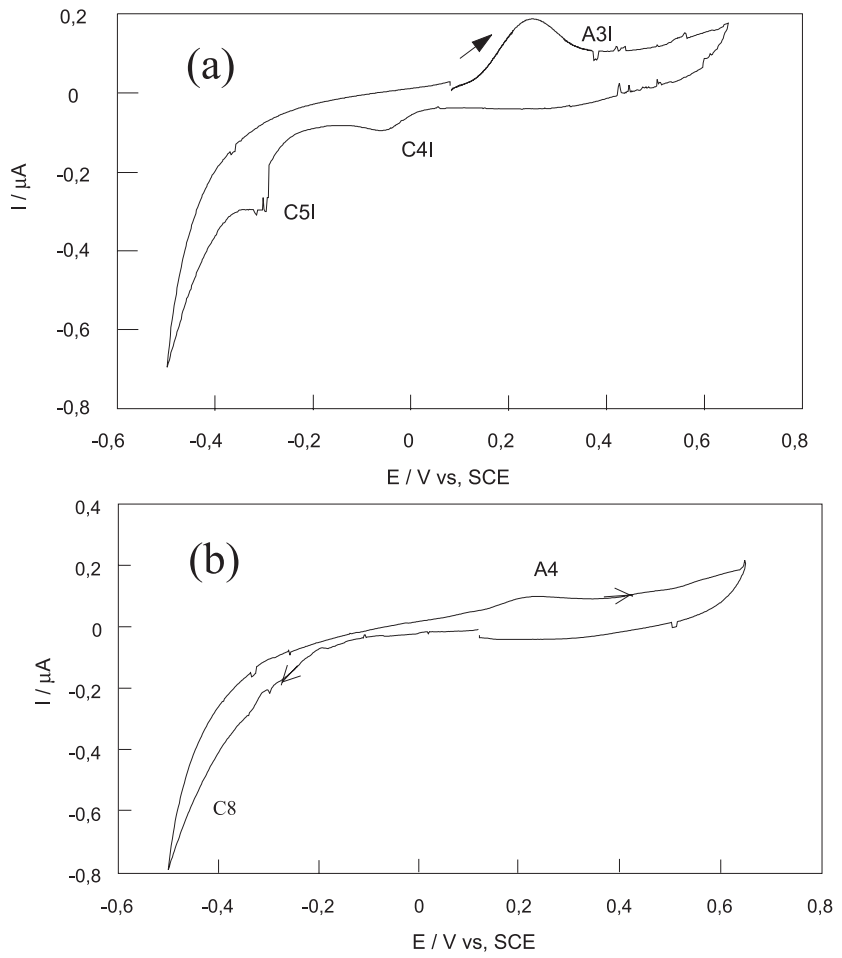

Figura 6. Voltamperograma de CPE-As $S_{2}$ (50:50\% peso) en el medio de cultivo M2, $v=20 \mathrm{mV} \mathrm{s}^{-1}$. El área del CPE-As $S_{2}$ fue de 0,0314 $\mathrm{cm}^{2}$. Barrido de potencial iniciado en dirección: (a) anódica y (b) catódica. Reimpresa da ref. 12, con permiso de The Electrochemical Society, Inc. 
que los procesos en $(\mathbf{C 4 I})$ y $(\mathbf{C 5 I})$ corresponden a la reducción de las especies formadas en el barrido directo, (A3I). Mientras que el proceso (C8) corresponde a la reducción del rejalgar. Las reacciones asociadas con los procesos descritos en los voltamperogramas de la Figura 6 fueron discutidos de la siguiente manera ${ }^{12}$ :

Procesos en (A3I):

corresponden a la oxidación del rejalgar

$\mathrm{As}_{2} \mathrm{~S}_{2}+14 \mathrm{H}_{2} \mathrm{O} \rightarrow 2 \mathrm{H}_{3} \mathrm{AsO}_{3}+2 \mathrm{SO}_{4}{ }^{2-}+22 \mathrm{H}^{+}+18 \mathrm{e}^{-}$

y a la ya discutida oxidación del ácido arsenioso $\left(\mathrm{H}_{3} \mathrm{AsO}_{3}\right)$ a ácido arsénico $\left(\mathrm{H}_{3} \mathrm{AsO}_{4}\right)$, Ecuación (9).

Proceso $(\mathbf{C 4 I})$ :

Reducción del ácido arsénico, inversa de la Ecuación (9)

Proceso $(\mathbf{C 5 I})$ :

Reducción directa de rejalgar, Ecuación (11)

Los resultados discutidos por Lázaro y colaboradores ${ }^{12}$, indicaron que el rejalgar $\left(\mathrm{As}_{2} \mathrm{~S}_{2}\right)$ se oxida en dos etapas, la primera a ácido arsenioso $\left(\mathrm{H}_{3} \mathrm{AsO}_{3}\right)$ y la segunda, es la oxidación del ácido arsenioso, en la interfase, a ácido arsénico $\left(\mathrm{H}_{3} \mathrm{AsO}_{4}\right)$. Estos productos fueron los mismos que los obtenidos en la oxidación del oropimente $\left(\mathrm{As}_{2} \mathrm{~S}_{3}\right)$. Por el contrario, los productos de reducción del $\mathrm{As}_{2} \mathrm{~S}_{2}$, fueron $\mathrm{As}^{0}$ y $\mathrm{H}_{2} \mathrm{~S}_{\text {(ac) }}$. Mientras que la reducción de $\mathrm{As}_{2} \mathrm{~S}_{3}$ sucedió en dos etapas: la reducción de $\mathrm{As}_{2} \mathrm{~S}_{3}$ a $\mathrm{As}_{2} \mathrm{~S}_{2}$ seguida de la reducción de $\mathrm{As}_{2} \mathrm{~S}_{2}$ a $\mathrm{As}^{0}$ y $\mathrm{H}_{2} \mathrm{~S}_{(\text {ac) }}$.

\section{Estudio electroquímico de la esfalerita}

A continuación se muestra un estudio electroquímico obtenido sobre un mineral poco estudiado como es la esfalerita ( $\mathrm{ZnS})$. La razón por la cual no existen reportados muchos trabajos respecto a este mineral es debido a que la esfalerita es uno de los minerales más resistivos $\left(10^{9}-10^{12} \Omega-\mathrm{cm}\right)$, propiedad por la cual es considerado como aislante. Por lo antes mencionado, los estudios electroquímicos sobre especímenes sólidos de $\mathrm{ZnS}$ no pueden ser llevados a cabo. No obstante, estos estudios sí pueden ser conducidos sobre CPE-mineral, debido a que la conductividad de este electrodo compuesto se ve mejorada debido a un fenómeno de percolación ${ }^{6}$.

Ahlberg y Asbjörnsson ${ }^{6}$, mostraron un estudio electroquímico sobre dos muestras de esfalerita que contenían 0,8 y 8,2\% peso de Fe dentro de su estructura. En ese estudio, estos mismos autores, utilizando CPE-esfalerita, demostraron que la esfalerita con bajo contenido en hierro es inactiva. En esta revisión se muestra un estudio electroquímico realizado por Cisneros-González y colaboradores $^{14,15}$ sobre un concentrado de flotación de esfalerita $(13,8 \%$ peso de $\mathrm{Fe}$ ) y sobre una esfalerita nativa que contenía $0,43 \%$ peso de Fe.

En la Figura 7 se muestran dos voltamperogramas trazados en la dirección positiva a partir del OCP a $100 \mathrm{mV} \mathrm{s}^{-1}$, sobre: (a) CPEconcentrado de esfalerita (13,8\% peso de $\mathrm{Fe})$ y (b) CPE-esfalerita natural $(0,43 \%$ peso de $\mathrm{Fe})$. Estos voltamperogramas fueron trazados en 1,0 $\mathrm{M} \mathrm{NaClO}_{4}$ a $\mathrm{pH}=2$, sin agitación de electrolito.

Del análisis de los voltamperogramas destaca que el concentrado de esfalerita (Figura 7a) presentó una mejor respuesta electroquímica respecto al obtenido sobre esfalerita natural, (Figura 7b). Esta diferencia pone en evidencia que el contenido de hierro como solución sólida, dentro de la esfalerita, es la responsable de obtener procesos de electrooxidación.

En un estudio cronoamperométrico y voltamperométrico cam-

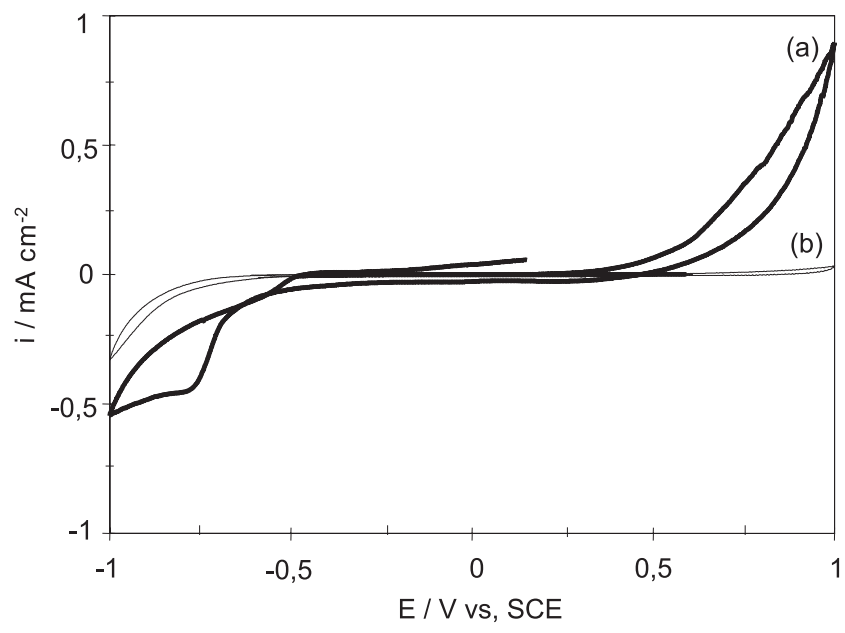

Figura 7. Voltamperograma cíclico típico obtenido en CPE- con diferentes muestras de esfalerita (40:60\% peso) en 1,0 $\mathrm{M} \mathrm{NaClO}_{4}$ a $\mathrm{pH}=2$. El área del CPE-esfalerita fue de $0,0314 \mathrm{~cm}^{2}$. El potencial de barrido fue iniciado en dirección positiva $\left(v=100 \mathrm{mV} \mathrm{s}^{-1}\right)$ : (a) concentrado de esfalerita $(13,8 \% \mathrm{Fe}$ en solución sólida); (b) esfalerita natural $(0,43 \% \text { Fe en solución sólida })^{15}$.

biando los potenciales de inversión, Cisneros-González y colaboradores ${ }^{15}$ mostraron las diferentes reacciones anódicas que toman lugar durante la oxidación de esfalerita como función del potencial.

$$
\begin{aligned}
& \mathrm{A} \mathrm{E} \leq 0,560 \mathrm{~V} \text { vs. SCE } \\
& \mathrm{ZnS} \rightarrow \mathrm{S}+\mathrm{Zn}^{2+}+2 \mathrm{e}^{-}
\end{aligned}
$$

A E $\geq 0,560 \mathrm{~V}$ vs. SCE

$$
\begin{aligned}
& 2 \mathrm{ZnS}+3 \mathrm{H}_{2} \mathrm{O} \rightarrow \mathrm{S}_{2} \mathrm{O}_{3}{ }^{2-}+2 \mathrm{Zn}^{2+}+6 \mathrm{H}^{+}+8 \mathrm{e}^{-} \\
& \mathrm{ZnS}+4 \mathrm{H}_{2} \mathrm{O} \rightarrow \mathrm{SO}_{4}{ }^{2-}+\mathrm{Zn}^{2+}+8 \mathrm{H}^{+}+8 \mathrm{e}^{-}
\end{aligned}
$$

Los resultados encontrados por Cisneros-González ${ }^{5}$ y colaboradores $^{14,15}$ mostraron las disolución anódica de esfalerita depende de la cantidad de hierro como solución sólida, donde una esfalerita que contenía $0,43 \%$ peso de Fe mostró ser inactiva; mientras que otra que contenía $13,8 \%$ peso de Fe permitió obtener reacciones electroquímicas dependientes del potencial externo. Es importante mencionar que a través del empleo de los CPE-esfalerita, como electrodos de prueba, fue posible estudiar a la esfalerita, lo cual es imposible empleando electrodos sólidos.

\section{Mecanismo de la disolución anódica de un concentrado complejo de cinc (que contiene $63,4 \% \mathrm{ZnS}, 20,1 \% \mathrm{FeS}_{2}, 5 \%$ $\mathrm{CuFeS}_{2}, 0,33 \% \mathrm{PbS}, 0,45 \% \mathrm{Cu}_{12} \mathrm{Sb}_{4} \mathrm{~S}_{13}$ y $0,4 \% \mathrm{FeAsS}$ ) en $\mathrm{H}_{2} \mathrm{SO}_{4}$}

En la actualidad, se tienen muchas minas complejas (no explotadas), donde los diferentes minerales se encuentran en similares concentraciones. Por lo tanto, debido a que los procesos de flotación no son selectivos, los concentrados de flotación suelen contener a los diferentes minerales de la mina explotada ${ }^{1}$, haciendo el procesamiento pirometalúrgico poco rentable. Por lo anterior, la ruta hidrometalúrgica ha sido considerada como una alternativa para el procesamiento de concentrados complejos ${ }^{1}$. Sin embargo, los estudios electroquímicos que se encuentran reportados para este tipo de concentrados son escasos.

En esta comunicación, se muestra un resumen del estudio de la disolución anódica de un concentrado complejo de cinc provenien- 
te de un proceso de flotación $\left(63,4 \% \mathrm{ZnS}, 20,1 \% \mathrm{FeS}_{2}, 5 \% \mathrm{CuFeS}_{2}\right.$, $0,33 \% \mathrm{PbS}, 0,45 \% \mathrm{Cu}_{12} \mathrm{Sb}_{4} \mathrm{~S}_{13}$ y $0,4 \% \mathrm{FeAsS}$ ), en $1,7 \mathrm{M} \mathrm{H}_{2} \mathrm{SO}_{4}{ }^{16}$.

En la Figura 8, se muestran dos voltamperogramas trazados en la dirección: (a) negativa y (b) positiva sobre CPE-concentrado de cinc en $1.7 \mathrm{M} \mathrm{H}_{2} \mathrm{SO}_{4}\left(v=100 \mathrm{mV} \mathrm{s}^{-1}\right)$. La comparación de estos voltamperogramas indicaron que los procesos $\left(\mathrm{C}_{1}{ }^{\prime}\right),\left(\mathrm{C}_{2}{ }^{\prime}\right),\left(\mathrm{C}_{3}{ }^{\prime}\right) \mathrm{y}$ $\left(\mathrm{C}_{4}{ }^{\prime}\right)$, están asociados con la reducción de los productos de oxidación formados en $\left(\mathrm{A}_{1}{ }^{\prime}\right)$. Para obtener información respecto a los minerales que se están oxidando en ( $\left.\mathrm{A}_{1}{ }^{\prime}\right)$, Nava et al. ${ }^{16}$ aplicaron pulsos anódicos de potencial, $\mathrm{E}_{\text {ap1 }}$, sobre CPE-concentrado de cinc durante $180 \mathrm{~s}$ en el intervalo comprendido entre $0,375 \leq \mathrm{E}_{\text {ap } 1} \leq$ $0,975 \mathrm{~V}$.

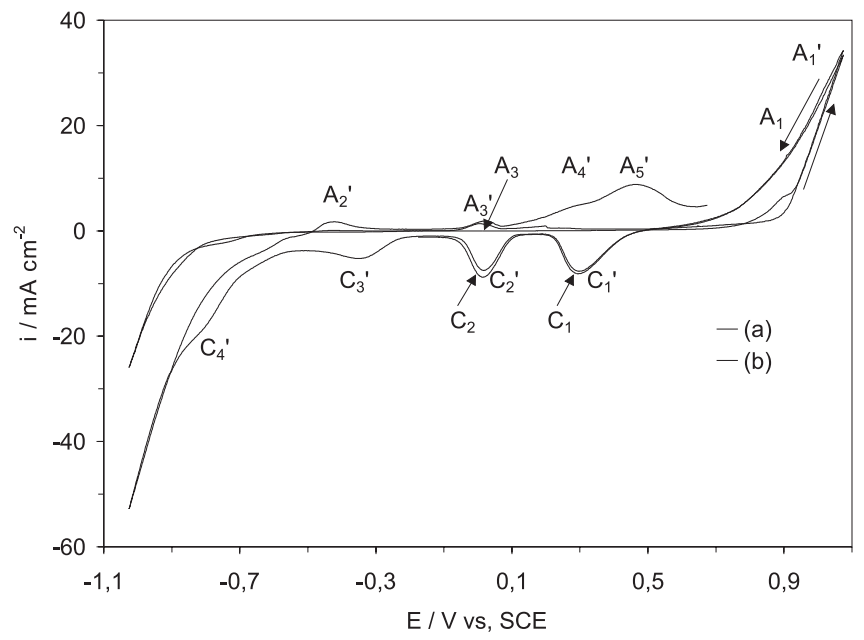

Figura 8. Voltamperogramas típicos obtenidos sobre CPE-concentrado complejo de cinc (80:20\% peso), en 1,7 $\mathrm{M} \mathrm{H}_{2} \mathrm{SO}_{4}\left(v=100 \mathrm{mV} \mathrm{s}^{-1}\right)$. El área del CPE-concentrado complejo fue de $0,0314 \mathrm{~cm}^{2}$. El barrido de potencial inició a partir del OCP (- 0,021 V vs. SCE) en dirección: (a) negativa, (b) positiva. Reimpresa da ref. 16, con permiso de The Electrochemical Society, Inc.

En la Figura 9 se muestra la curva Qa vs. $\mathrm{E}_{\text {ap1 }}$ construida, a partir de la integración de la carga de los cronoamperogramas, a cada $\mathrm{E}_{\text {ap1 }}$. En esta Figura son mostradas cuatro zonas de potencial, donde la variación de Qa presenta una tendencia similar como función de $\mathrm{E}_{\text {ap1 }}$. Nava y colaboradores ${ }^{16}$ discutieron que estas tenden-

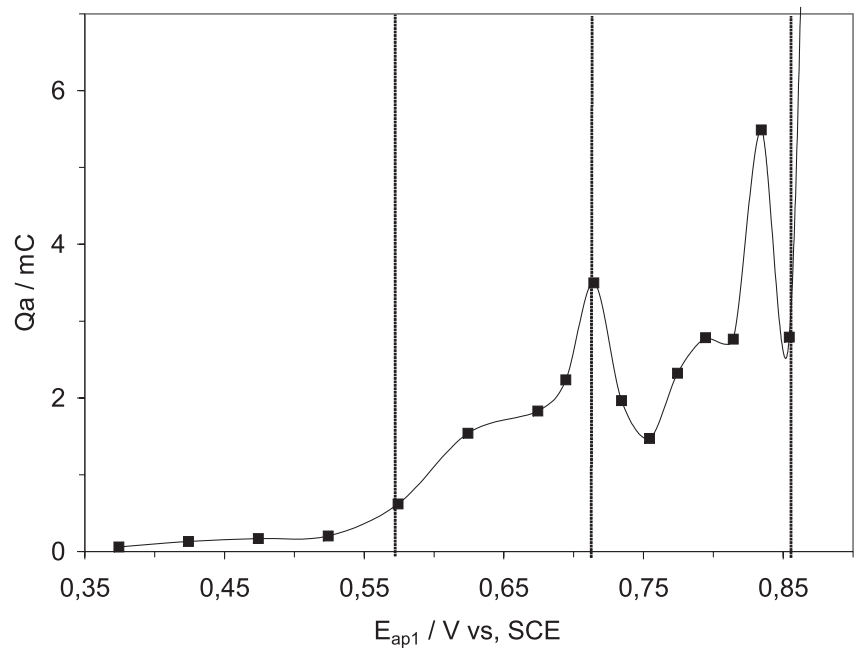

Figura 9. Cargas anódicas (Qa) como función del pulso de potencial anódico $\left(E_{\text {apl }}\right)$, evaluadas a partir de los pulsos potenciostáticos aplicados sobre CPEconcentrado complejo de cinc, en 1,7 $\mathrm{M} \mathrm{H}_{2} \mathrm{SO}_{4}$. Reimpresa da ref. 16, con permiso de The Electrochemical Society, Inc. cias podrían corresponder a la oxidación de los diferentes minerales presentes en el concentrado. Para comprobar esto, estos mismos autores, analizaron a los diferentes metales contenidos en el electrolito después de cada pulso de potencial, $\mathrm{E}_{\text {apl }}$, impuesto al CPE-concentrado de cinc.

Para el análisis de los diferentes metales contenidos en el licor (después de la imposición de $\mathrm{E}_{\mathrm{ap} 1}$ sobre el CPE-concentrado de cinc), Nava y colaboradores ${ }^{16}$ emplearon la técnica de redisolución anódica, utilizando electrodos de película fina de mercurio. De las voltamperometrías de redisolución anódica se evaluaron la corrientes de los picos asociados a $\mathrm{Zn}, \mathrm{Cd}, \mathrm{Pb}$ y $\mathrm{Cu}$. La magnitud de las corrientes de los picos de redisolución anódicos $\left(\mathrm{I}_{\text {pra }}\right)$ fue graficada para cada metal como función del pulso de oxidación, $\mathrm{E}_{\text {ap1 }}$, (Figura 10).

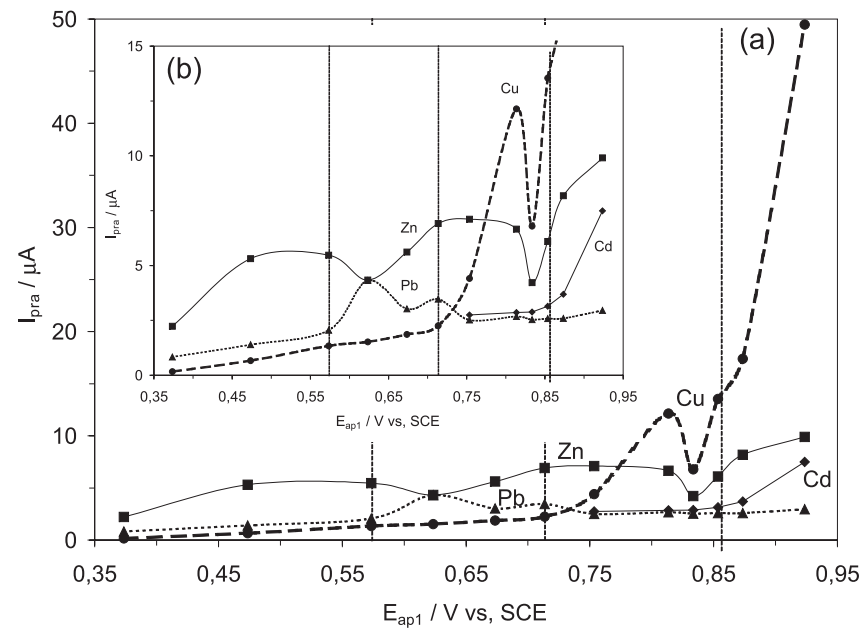

Figura 10. (a) Corrientes de los picos de redisolución anódica $\left(I_{p r a}\right)$, obtenidas en el licor que contiene a los diferentes metales electrodisueltos después de que el CPE-concentrado complejo de cinc fue oxidado a los diferentes pulsos anódicos, $\left(E_{a p 1}\right)$. (b) Es una ampliación de $(a)$. Las $I_{\text {pra }}$ fueron evaluadas de los voltamperogramas de pulso diferencial de redisolución anódica, utilizando electrodos de película fina de mercurio soportadas en disco de carbono vítreo. El disco de carbón vítreo tenía un área de 0,1963 cm². Reimpresa da ref. 16, con permiso de The Electrochemical Society, Inc.

De la Figura 10b, fue posible distinguir que la electrodisolución de cinc fue progresiva en todo el dominio del pulso de potencial estudiado, $0,375 \leq \mathrm{E}_{\mathrm{ap} 1} \leq 0,925 \mathrm{~V}$. El comportamiento de la disolución de plomo, fue similar a la obtenida para cinc, sólo que las $\mathrm{I}_{\text {pra }}$ fueron menores. $\mathrm{A}_{\mathrm{ap1}} \geq 0,855 \mathrm{~V}$, la redisolución anódica de $\mathrm{Cu}$ creció abruptamente con $\mathrm{E}_{\text {ap1 }}$, Figura 10a. La detección de cadmio en el licor comenzó a darse a partir de $\mathrm{E}_{\text {ap1 }}>0,755 \mathrm{~V}$, Figura $10 \mathrm{~b}$. Es importante mencionar que el concentrado complejo de cinc, contenía $\pm 0,01 \%$ de cadmio como solución sólida (Zn, Cd)S. A través del análisis en el licor resultante, Nava y colaboradores ${ }^{16}$ distinguieron a los diferentes metales que dejan el concentrado complejo como función del potencial de oxidación, $\mathrm{E}_{\text {ap1 }}$.

Por otro lado, estos mismos autores complementaron este estudio caracterizando la superficie modificada del CPE-concentrado de cinc electro-oxidado, después de cada $\mathrm{E}_{\text {ap1 }}$. Esta caracterización se llevó a cabo en electrolito fresco $\left(1,7 \mathrm{M} \mathrm{H}_{2} \mathrm{SO}_{4}\right)$ libre de productos de disolución.

En este resumen sólo se muestra la caracterización de los productos de oxidación formados en el intervalo de $0,575 \leq \mathrm{E}_{\text {ap } 1} \leq$ $0,715 \mathrm{~V}$. En los voltamperogramas trazados después de $\mathrm{E}_{\text {ap } 1}$ de 0,675 y $0,715 \mathrm{~V}$, (Figura $11 \mathrm{~b}-\mathrm{c})$, se detectó el par $\left(\mathrm{C}_{1}\right) /\left(\mathrm{A}_{5}\right)$, el cuál no fue observado sobre concentrado fresco, (Figura 11a). El par $\left(\mathrm{C}_{1}\right) /\left(\mathrm{A}_{5}\right)$ 


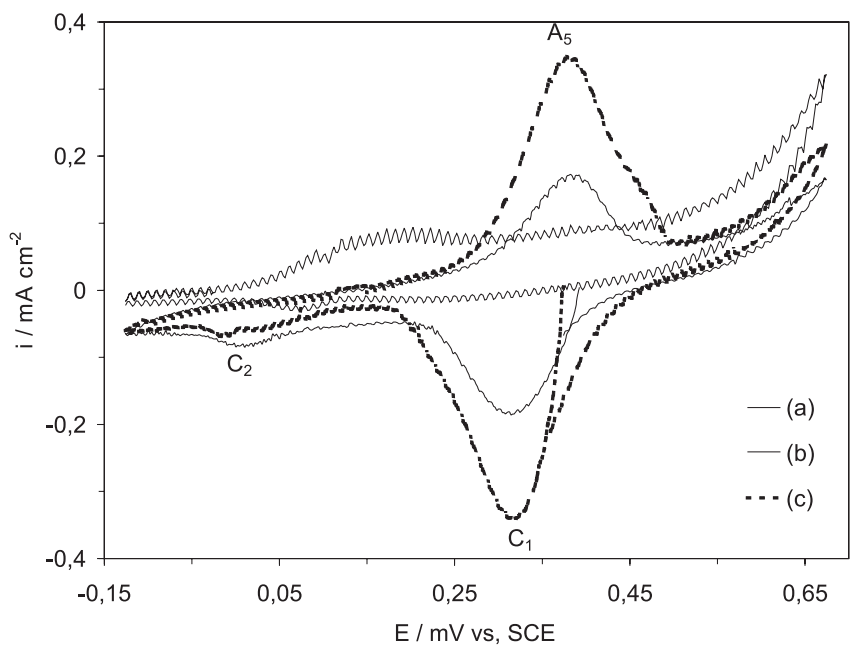

Figura 11. Voltamperogramas típicos obtenidos sobre la superficie modificada de CPE-concentrado complejo de cinc, en electrolito recién preparado, 1,7 M $\mathrm{H}_{2} \mathrm{SO}_{4},\left(v=100 \mathrm{mV} \mathrm{s}^{-1}\right)$. El área del CPE-concentrado complejo modificado fue de $0,0314 \mathrm{~cm}^{2}$. El electrodo modificado fue enjuagado con agua desionizada y sumergido en electrolito recién preparado, donde reposó $30 \mathrm{~s}$ antes del trazado del voltamperograma. El CPE-concentrado complejo de cinc fue previamente modificado por pulsos de potencial de oxidación, $E_{a p 1}$, a: (b) 675, (c) $715 \mathrm{mV}$ vs. SCE, por $180 \mathrm{~s}$ en cada $E_{a p 1}$, y estos fueron comparados con uno obtenido sobre concentrado complejo de cinc fresco, (a). Todos los voltamperogramas fueron iniciados en la dirección negativa. Reimpresa da ref. 16, con permiso de The Electrochemical Society, Inc.

fue más importante conforme el pulso de potencial $\left(\mathrm{E}_{\text {ap1 }}\right)$ fue mayor, (Figura 11c). Es importante mencionar que en los voltamperogramas obtenidos sobre la superficie modificada (Figura 11b-c), también apareció un proceso de reducción $\left(\mathrm{C}_{2}\right)$.

Nava y colaboradores ${ }^{16}$ discutieron que las reacciones en $\left(\mathrm{C}_{1}\right) /$ $\left(\mathrm{A}_{5}\right)$, pueden estar asociadas con la reducción-oxidación de un polisulfuro de cobre y hierro no estequiométrico:

$\mathrm{Cu}_{1-\mathrm{x}} \mathrm{Fe}_{1-\mathrm{y}} \mathrm{S}_{2-\mathrm{z}}+2 \mathrm{bH}^{+}+2(\mathrm{~b}-\mathrm{a}) \mathrm{e}^{-} \Leftrightarrow \mathrm{Cu}_{1-\mathrm{x}} \mathrm{Fe}_{1-\mathrm{y}-\mathrm{a}} \mathrm{S}_{2-\mathrm{z}-\mathrm{b}}+\mathrm{aFe}^{2+}+\mathrm{bH}_{2} \mathrm{~S}$

Esta reacción fue propuesta por Nava y colaboradores ${ }^{16}$ considerando que diversos investigadores ${ }^{17,18}$ han informado que la oxi- dación de calcopirita a $\mathrm{E}>0,575 \mathrm{~V}$, forma un sulfuro de cobre y hierro no estequiométrico:

$\mathrm{CuFeS}_{2} \Leftrightarrow \mathrm{Cu}_{1-\mathrm{x}} \mathrm{Fe}_{1-\mathrm{y}} \mathrm{S}_{2-\mathrm{z}}+\mathrm{xCu}^{2+}+\mathrm{yFe}^{2+}+\mathrm{zS}+2(\mathrm{x}+\mathrm{y}) \mathrm{e}^{-}$

Además, la detección de cobre en el licor soporta esta discusión, (Figura 10a).

Gardener y Woods ${ }^{19}$ mostraron que el proceso de reducción $\left(\mathrm{C}_{2}\right)$ es la reducción de un hidróxido de hierro proveniente de la disolución anódica de la calcopirita:

$\mathrm{Fe}(\mathrm{OH})_{3}+3 \mathrm{H}^{+}+1 \mathrm{e}^{-} \Leftrightarrow \mathrm{Fe}^{2+}+3 \mathrm{H}_{2} \mathrm{O}$

De igual manera, se estudió la oxidación del concentrado complejo de cinc en todo el dominio de potencial estudiado, $0,375 \leq$ $\mathrm{E}_{\text {ap1 }} \leq 0,925 \mathrm{~V}$. En la Tabla 1 se resumen las reacciones electroquímicas que toman lugar durante la oxidación del concentrado complejo de cinc a potencial constante, $\mathrm{E}_{\text {apl }}$, en $1.7 \mathrm{M} \mathrm{H}_{2} \mathrm{SO}_{4}{ }^{16}$.

La estrategia electroquímica propuesta por Nava y colaboradores ${ }^{16}$ permitió distinguir cómo los diferentes sulfuros metálicos, contenidos en el concentrado complejo de cinc, fueron oxidados como función del potencial. De esta manera, se puso en evidencia la conveniencia del empleo de los CPE-mineral para el estudio de concentrados complejos.

\section{Estudio electroquímico de diferentes minerales que contienen plata}

En esta comunicación, se muestra un estudio electroquímico, sobre dos muestras de minerales que contienen plata de diferentes orígenes (Las Torres y Real del Monte). Este estudio permitió identificar cual de las diferentes fases de plata (diferentes soluciones sólidas), son refractarias a la lixiviación por cianuración ${ }^{20}$,

En la Tabla $2^{21}$, se muestra el contenido de los metales presentes en 4 diferentes minerales, (Las Torres, Real del Monte, Fresnillo y Echo Bay). Las dos muestras de interés en esta revisión son Las Torres y Real del Monte, las cuales mostraron una gran diferencia en las fases de plata presentes. Para el concentrado de Real del Monte, sólo se observó acantita $\left(\mathrm{Ag}_{2} \mathrm{~S}\right)$. En el caso de Las Torres, las fases de plata encontradas fueron cuatro (aguilarita $\left(\mathrm{Ag}_{4} \mathrm{SeS}\right) 80,4 \%$, freibergita $(\mathrm{Cu}, \mathrm{Ag}, \mathrm{Zn}, \mathrm{Fe})_{12} \mathrm{SbS}_{13} 4,3 \%$, polibasita $\left(9 \mathrm{Ag}_{2} \mathrm{~S}_{2} \mathrm{Sb}_{2} \mathrm{~S}_{3}\right) 3,9 \%$ y hessita $\left(\mathrm{Ag}_{2} \mathrm{Te}\right) 1,4 \%$ en abundancia relativa). Luna-Sánchez y colaboradores ${ }^{20}$ tomaron como modelos de

Tabla 1. Reacciones electroquímicas que toman lugar durante la oxidación del concentrado de cinc a potencial constante, $\mathrm{E}_{\text {ap1 }}$, en $1.7 \mathrm{M}$ $\mathrm{H}_{2} \mathrm{SO}_{4}$. Reimpresa da ref. 16, con permiso de The Electrochemical Society, Inc.

\begin{tabular}{|c|c|}
\hline $\begin{array}{l}\text { Intervalo de potencial/ } \\
\text { (V vs. SCE) }\end{array}$ & Reacciones Electroquímicas \\
\hline $0,375 \leq \mathrm{E}_{\text {ap } 1}<0,575$ & $\begin{array}{l}\mathrm{ZnS} \rightarrow \mathrm{Zn}^{2+}+\mathrm{S}+2 \mathrm{e}^{-} \\
\mathrm{PbS} \rightarrow \mathrm{Pb}^{2+}+\mathrm{S}+2 \mathrm{e}^{-}\end{array}$ \\
\hline $\begin{array}{l}0,575 \leq \mathrm{E}_{\text {ap1 }}<0,715 \\
\mathrm{E}_{\text {ap } 1} \geq 0,575 \\
0,575<\mathrm{E}_{\text {ap1 }} \leq 0,715\end{array}$ & $\begin{array}{l}\mathrm{ZnS}+4 \mathrm{H}_{2} \mathrm{O} \rightarrow \mathrm{Zn}^{2+}+\mathrm{SO}_{4}^{2-}+8 \mathrm{H}^{+}+8 \mathrm{e}^{-} \\
\mathrm{PbS}+4 \mathrm{H}_{2} \mathrm{O} \rightarrow \mathrm{Pb}^{2+}+\mathrm{SO}_{4}^{2-}+8 \mathrm{H}^{+}+8 \mathrm{e}^{-} \\
\mathrm{CuFeS}_{2} \Leftrightarrow \mathrm{Cu}_{1-\mathrm{Fe}} \mathrm{Fe}_{1-\mathrm{S}} \mathrm{S}_{2-\mathrm{z}}+x \mathrm{Cu}^{2+}+y \mathrm{Fe}^{2+}+z \mathrm{~S}+2(x+y) \mathrm{e}^{-} \\
\mathrm{Fe}^{2+}+3 \mathrm{H}_{2} \mathrm{O} \Leftrightarrow \mathrm{Fe}(\mathrm{OH})_{3}+3 \mathrm{H}^{+}+1 \mathrm{e}^{-}\end{array}$ \\
\hline $\begin{array}{l}0,715 \leq \mathrm{E}_{\mathrm{ap} 1} \leq 0,855 \\
0,715 \leq \mathrm{E}_{\mathrm{ap} 1} \leq 0,815 \\
0,835 \leq \mathrm{E}_{\mathrm{ap} 1} \leq 0,855\end{array}$ & $\begin{array}{l}\text { Oxidación de Calcopirita a sulfuro de cobre y hierro no estequiométrico } \\
\mathrm{CuFeS}_{2} \Leftrightarrow \mathrm{CuS}+\mathrm{Fe}^{2+}+2 \mathrm{~S}^{0}+2 \mathrm{e}^{-} \text {y reacciones de oxidación de galena y esfalerita a } \mathrm{E}_{\text {ap1 }} \geq 0,715 \mathrm{~V}\end{array}$ \\
\hline $\begin{array}{l}0,855<\mathrm{E}_{\text {ap1 }} \leq 0,975 \\
0,855<\mathrm{E}_{\text {ap1 }} \leq 0,875 \\
0,875<\mathrm{E}_{\text {ap1 }} \leq 0,975\end{array}$ & $\begin{array}{l}\mathrm{FeS}_{2} \Leftrightarrow \mathrm{Fe}_{1-\mathrm{x}} \mathrm{S}_{2-\mathrm{y}}+\mathrm{xFe}^{3+}+\mathrm{yS}+3 \mathrm{xe}^{-} \\
\mathrm{FeS}_{2}+8 \mathrm{H}_{2} \mathrm{O} \Leftrightarrow \mathrm{Fe}^{2+}+2 \mathrm{SO}_{4}^{2-}+16 \mathrm{H}^{+}+14 \mathrm{e}^{-} \text {y reacciones de oxidación de galena, esfalerita y calcopirita a } \\
\mathrm{E}_{\text {ap } 1}>0,855 \mathrm{~V}\end{array}$ \\
\hline
\end{tabular}


comparación los minerales de Echo Bay $98 \%$ Ag 2 y y Fresnillo $97 \%$ pirita. Por razones prácticas de nomenclatura, estos mismos autores les llamaron acantita y pirita respectivamente.

Tabla 2. Porcentaje de metal (\%) presente en cada concentrado y mineral de plata $^{21}$

\begin{tabular}{lcccc}
\hline Metal & Las Torres & Real del Monte & Fresnillo & Echo Bay \\
\hline Plata & 1,23 & 1,20 & n.a & 86,27 \\
Cobre & 0,93 & 0,27 & 0,62 & 0,87 \\
Plomo & 0,47 & 0,92 & n.a & 0,0 \\
Hierro** $_{\text {Zinc }}^{*}$ & 37,95 & 21,40 & 47,80 & 2,54 \\
Oro & 0,66 & 0,47 & n.a & 0,0 \\
& $80,80^{*}$ & $7,25^{*}$ & n.a & 0,0 \\
\hline
\end{tabular}

${ }^{*} \mathrm{~g} \mathrm{Au} /$ ton mineral; n.a no analizada. ${ }^{* *}$ El hierro se encuentra en forma de pirita.

En la Figura 12, se presentan voltamperogramas obtenidos sobre la muestra de Real del Monte (Figura 12a ii) y Las Torres (Figura $12 \mathrm{~b}$ iii), con respecto a acantita (Figura $12 \mathrm{a}$ i y $12 \mathrm{~b}$ i). En el barri-
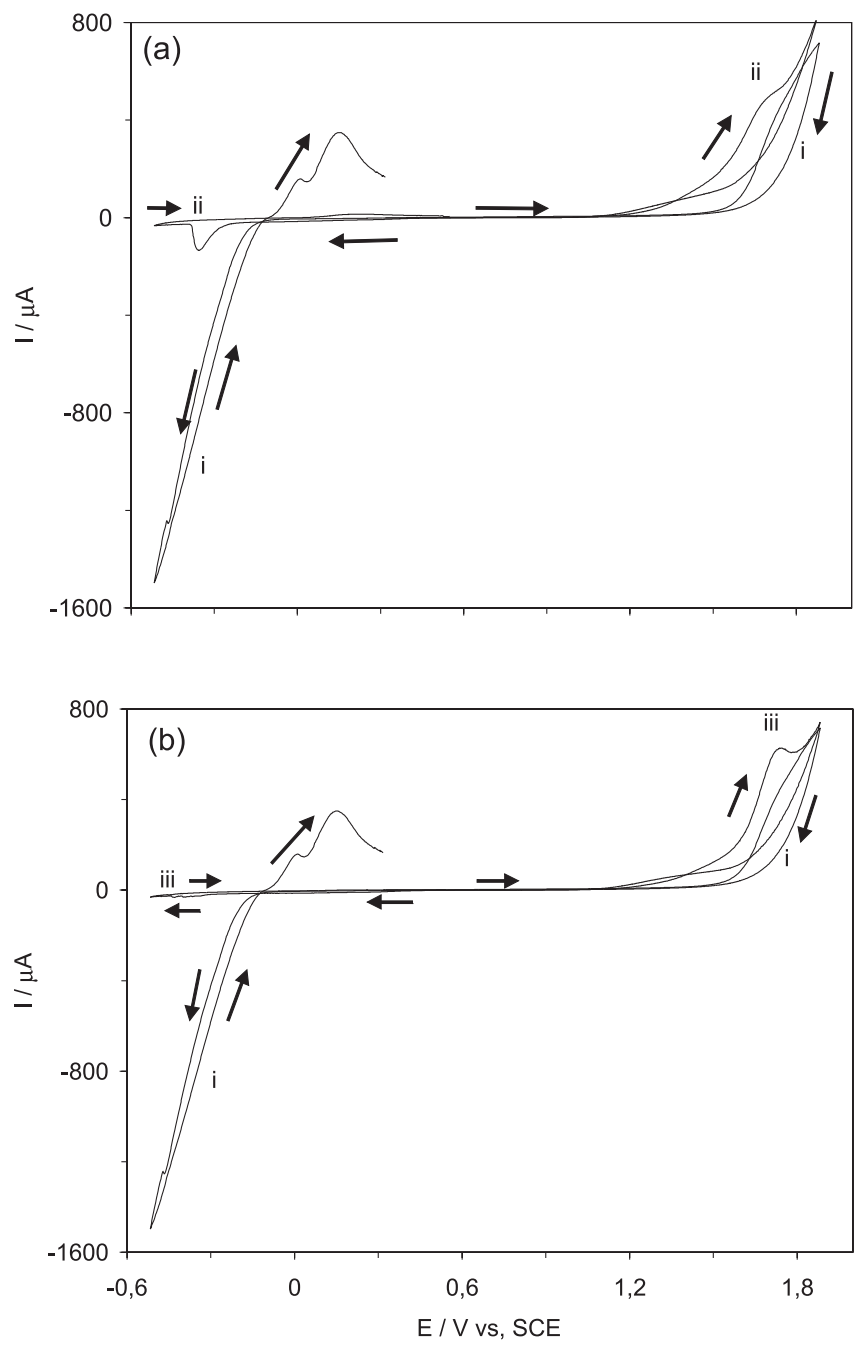

Figura 12. Voltamperogramas típicos obtenidos sobre CPE-mineral (70:30\% peso) en 0,3 M NaCN a pH =10,60. Barrido de potencial iniciado en la dirección positiva a partir del potencial de circuito abierto $\left(v=25 \mathrm{mV} \mathrm{s}^{-1}\right)$. El área del CPE-mineral fue de 0,0314 $\mathrm{cm}^{2}$. (a ii) Real del Monte, (b iii) Las Torres. Línea fina, Acantita (i en a y b). Reimpresa da ref. 20, con permiso de Springer Science and Bussines Media do directo sobre ambos concentrados (Figura 12a ii y Figura 12b iii), la oxidación comienza a potenciales menores que $1.2 \mathrm{~V}$, lo cual es menor que el potencial en donde la acantita comienza a oxidarse (Figura 12a i y Figura 12b i). Esto es debido a que estos procesos se encuentran ocultos por la oxidación masiva de pirita contenida en ambos concentrados. En el barrido inverso (para Real del Monte) se detectó un proceso de reducción a -0,3 V vs. SCE (Figura 12a ii); el cual fue muy difícil de distinguir para Las Torres (Figura 12b iii).

En la Figura 13, se muestra una ampliación de la zona catódica de los voltamperogramas típicos obtenidos sobre CPE-mineral: Pirita (i), (ii) Real del Monte y (iii) Las Torres. Es importante mencionar que todos los voltamperogramas mostrados en esta Figura fueron iniciados en la dirección positiva. Cuando el barrido de potencial es aplicado, la aparición de un pequeño pico I fue asociado a la reducción de especies de hierro formadas durante el barrido directo. Por otro lado, cuando el barrido de potencial alcanzó valores más negativos se detectó un segundo pico II (Figura 13 ii y iii), el cual corresponde a la reducción de iones plata, liberados en el barrido directo. Con respecto a Las Torres (Figura 13 iii), la cantidad de plata liberada, durante el barrido directo fue menor que el obtenido por Real del Monte, (Figura 13 ii). Este comportamiento indica que la plata contenida en Las Torres es refractaria, es decir que es poco susceptible a la oxidación.

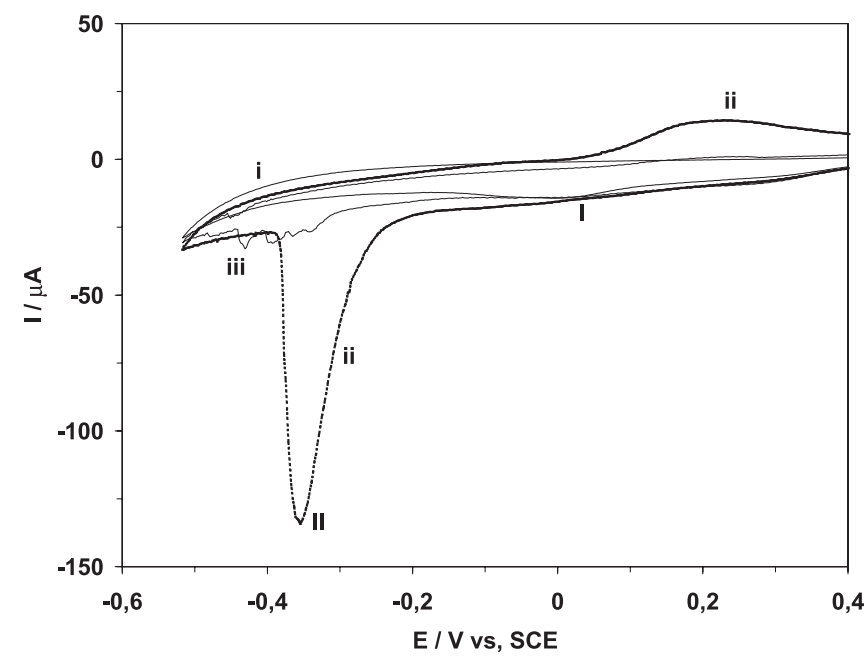

Figura 13. Zona catódica amplificada de los voltamperogramas típicos obtenidos sobre CPE-mineral (70:30\% peso) en 0,3 M NaCN a pH =10,60. Barrido de potencial iniciado en la dirección positiva a partir del potencial de circuito abierto $\left(v=25 \mathrm{mV} \mathrm{s}^{-1}\right)$. El área del CPE-mineral fue de 0,0314 $\mathrm{cm}^{2}$. Pirita (i), (ii) Real del Monte y (iii) Las Torres. Reimpresa da ref. 20, con permiso de Springer Science and Bussines Media

Por otro lado, Luna-Sánchez y colaboradores ${ }^{20}$ desarrollaron estudios de lixiviación (cianuración en presencia de $\mathrm{O}_{2}$, durante $8 \mathrm{~h}$, en ambos concentrados (Las Torres y Real del Monte), donde encontraron que la oxidación del mineral de Real del Monte fue más cuantitativa que en Las Torres. De esta manera, Luna-Sánchez y colaboradores ${ }^{20}$ mostraron la conveniencia del empleo de técnicas electroquímicas empleando CPE-mineral, para una rápida evaluación de las propiedades refractarias de diferentes concentrados minerales que contienen plata.

\section{CONCLUSIONES}

En esta comunicación se mostró la potencialidad de las técnicas electroquímicas y de los electrodos de pasta de carbono-mine- 
ral (CPE-mineral), en el estudio de los mecanismos de disolución de diferentes minerales. Se mostró una estrategia voltamperométrica que permitió encontrar el mecanismo de disolución de un concentrado de galena ( $\mathrm{PbS}$, altamente puro) proveniente de un proceso de flotación. El segundo estudio fue la disolución anódica dos minerales de fácil alteración, poco estudiados, como son el rejalgar $\left(\mathrm{As}_{2} \mathrm{~S}_{2}\right)$ y el oropimente $\left(\mathrm{As}_{2} \mathrm{~S}_{3}\right)$. El tercer estudio mostró el efecto del hierro, como solución sólida, sobre la actividad electroquímica de un mineral poco conductor ( $\mathrm{Zn}, \mathrm{Fe},) \mathrm{S}$. Por otro lado, se mostró una estrategia más elaborada que permitió encontrar el mecanismo de disolución de un concentrado complejo de cinc $(63,4 \% \mathrm{ZnS}$, $20,1 \% \mathrm{FeS}_{2}, 5 \% \mathrm{CuFeS}_{2}, 0,33 \% \mathrm{PbS}, 0,45 \% \mathrm{Cu}_{12} \mathrm{Sb}_{4} \mathrm{~S}_{13}$ y $0,4 \%$ FeAsS), mostrando como los diferentes minerales son oxidados como función del potencial. Finalmente, se presentó un método electroquímico que permitió identificar las propiedades refractarias a la lixiviación de diferentes fases de plata contenidas en concentrados minerales, de una manera rápida y efectiva respecto a las técnicas tradicionales.

\section{REFERENCIAS}

1. Dutrizac, J. E.; Hydrometallurgy 1992, $29,1$.

2. Bauer, D.; Gaillochet, M. P.; Electrochim. Acta 1974, 19, 597.

3. Lamache, M.; Electrochim. Acta 1979, 24, 79.

4. Gerlach, J.; Küzeci, K.; Hydrometallurgy 1983, 11, 345.
5. Ahlberg, E.; Asbjornsson, J.; Hydrometallurgy 1993, 34, 171

6. Ahlberg, E.; Asbjornsson, J.; Hydrometallurgy 1994, 36, 19.

7. Nava, J. L.; Oropeza, M. T.; González, I.; Electrochim. Acta 2002, 47, 1513.

8. Bard, A. J.; Parsons, R.; Jordan R.; Standard Potentials in Aqueous Solutions (IUPAC), Marcel Decker: New York, 1985.

9. Pourbaix, M.; Atlas of Electrochemical Equilibrium in Aqueous Solutions, Pergamon Press: Oxford, 1977.

10. Paul, R. L.; Nicol, M. J.; Diggle, J. W.; Saunders, A. P.; Electrochim. Acta 1978, 23, 625.

11. Cisneros-González, I.; Oropeza-Guzmán, M. T.; González, I.; Electrochim. Acta 2000, 17, 2729.

12. Lázaro, I.; González, I.; Cruz, R.; Monroy, M.; J. Electrochem. Soc. 1997, $12,4128$.

13. Cruz, R.; Lázaro, I.; Rodriguez, J. M.; Monroy, M.; González, I.; Hydrometallurgy 1997, 46, 303.

14. Cisneros-González, I.; Oropeza-Guzmán, M. T.; González, I. En Electrochemistry in Mineral and Metal Processing V; Woods, R.; Doyle, F. M., eds.; The Electrochemistry Society: New Jersey, 2000.

15. Cisneros-González, I.; Tesis de Doctorado; Universidad Autónoma Metropolitana-Iztapalapa, México, 2001.

16. Nava, J. L.; Oropeza, M. T.; González, I.; J. Electrochem. Soc. 2004, 151, B387.

17. Lázaro, I.; Martínez-Medina, N.; Rodriguez, I.; Arce, E.; González, I.; Hydrometallurgy 1995, 38, 277.

18. Arce, E.; González, I.; Int. J. Miner. Process. 2002, 67, 17.

19. Gardener, J. R.; Woods, R.; Int. J. Miner. Process. 1979, 6, 1.

20. Luna-Sánchez, R. M.; González, I.; Lapidus, G. T.; J. Appl. Electrochem. 2002, 32, 1157.

21. Luna-Sánchez, R. M.; Tesis de Doctorado; Universidad Autónoma Metropolitana-Iztapalapa, México, 2003. 